\title{
Atividade antifúngica dos óleos essenciais de sassafrás (Ocotea odorifera Vell.) e alecrim (Rosmarinus officinalis L.) sobre o gênero Candida
}

\author{
CASTRO, R.D. ${ }^{1 *}$; LIMA, E.O. ${ }^{2}$ \\ ${ }^{1}$ Universidade Federal da Paraíba, Departamento de Clínica e Odontologia Social, Campus Universitário I, Cidade \\ Universitária, CEP:58059-900, João Pessoa-Brasil *ricardodiasdecastro@yahoo.com.br 2Universidade Federal \\ da Paraíba, Departamento de Ciências Farmacêuticas, Campus Universitário I, Cidade Universitária, CEP: 58059- \\ 900, João Pessoa-Brasil
}

\begin{abstract}
RESUMO: Este estudo objetivou avaliar, in vitro, a atividade antifúngica dos óleos essenciais de Ocotea odorifera (Vellozo) Rohwer (Sassafrás) e Rosmarinus officinalis L. (Alecrim) sobre cepas de Candida albicans e C. tropicalis, envolvidas com infecções da cavidade oral. Para tanto, 16 cepas de Candida de origem clínica e de referência foram utilizadas para determinação da concentração inibitória mínima (CIM), utilizando a técnica da microdiluição. Miconazol e nistatina foram utilizados como controles positivos. Observou-se discreta atividade antifúngica de ambos os óleos, com CIM de 2,5 mg mL-1 para sassafrás e CIM de $5 \mathrm{mg} \mathrm{mL}^{-1}$ para alecrim em $68 \% \mathrm{e}$ $81 \%$ das cepas avaliadas, respectivamente. Todas as cepas de Candida mostraram-se sensíveis ao miconazol e nistatina. A partir dos resultados obtidos, foi possível concluir que os óleos essenciais de $O$. odorifera Vell. e $R$. officinalis $L$. apresentam fraca atividade sobre cepas de $C$. albicans e $C$. tropicalis envolvidas em infecções da cavidade oral.
\end{abstract}

Palavras-Chave: Rosmarinus officinalis L., Ocotea odorifera, candidíase bucal, Candida

\begin{abstract}
Antifungal activity of Brazilian sassafras (Ocotea odorifera Vell.) and rosemary (Rosmarinus officinalis L.) essential oils against the genus Candida. This study aimed to evaluate the in vitro antifungal activity of essential oils from Ocotea odorifera Vell. (Brazilian sassafras) and Rosmarinus officinalis L. (rosemary) against Candida albicans and C. tropicalis strains, both involved in oral cavity infections. Thus, 16 Candida strains from clinical origin and standards were used to determine the minimum inhibitory concentration (MIC), using the microdilution technique. Miconazole and nystatin were used as positive controls. A slight antifungal

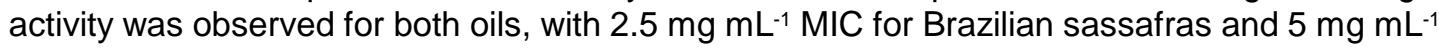
MIC for rosemary in 68 and $81 \%$ strains, respectively. All Candida strains were sensitive to miconazole and nystatin. In conclusion, essential oils from $O$. odorifera Vell. and $R$. officinalis $L$. had weak activity against $C$. albicans and $C$. tropicalis strains involved in oral cavity infections.
\end{abstract}

Key words: Rosmarinus officinalis, Ocotea odorifera, oral candidiasis, Candida

\section{INTRODUÇÃO}

Candida albicans e C. tropicalis têm sido apontadas como as principais espécies envolvidas em infecções fúngicas da cavidade oral e possuem como principal fator de virulência a capacidade de adesão aos tecidos bucais e formação de biofilmes (Thein et al., 2006). Tais infecções, conhecidas como candidíase ou candidose oral, estomatite eritematosa ou estomatite protética (quando associada ao uso de prótese dentária), constituem um frequente problema a ser enfrentado pelo cirurgião-dentista.
Indivíduos imunodeprimidos constituem o grupo de maior risco para o desenvolvimento da doença, tendo em vista o caráter oportunista dos microrganismos envolvidos. Porém, como descrito anteriormente, usuários de aparelhos protéticos, principalmente totais, apresentam com frequência lesões fúngicas na mucosa adjacente à resina acrílica, material utilizado para confecção desses aparelhos, que representa uma superfície susceptível para retenção de espécies de Candida e, consequentemente,

Recebido para publicação em 21/09/2009

Aceito para publicação em 08/04/2010

Rev. Bras. PI. Med., Botucatu, v.13, n.2, p.203-208, 2011. 
formação de biofilme (Henriques et al., 2004; Ramage et al., 2005).

Mecanismos moleculares possivelmente envolvidos na patogenicidade de espécies de Candida são alvos de pesquisas visando à elaboração de fármacos que apresentem maior seletividade e, consequentemente, menos efeitos indesejáveis (Orozco et al., 2000; Monge et al., 2006; Müller et al., 2007). Atualmente, os agentes disponíveis para tratamento de infecções fúngicas da cavidade oral, caracterizadas como superficiais, são representados pelos poliênicos (anfotericina B, nistatina, entre outros) e azólicos (fluconazol, itraconazol, miconazol, cetoconazol, entre outros), sendo estes últimos os eleitos em primeira instância para tratamento dessas doenças e são geralmente fungistáticos (Wingeter et al., 2007).

Diante das limitações de uso desses antifúngicos sintéticos, evidenciadas pelo aumento da resistência pelos microrganismos, bem como pelas reações indesejadas apresentadas pelos usuários, novos agentes são propostos na tentativa de minimizar tais ocorrências. Nesse sentido, considerando a ampla atividade biológica apresentada pelos produtos de origem natural, óleos essenciais obtidos a partir da $O$. odorifera Vell. e $R$. officinalis $L$. têm sido investigados para determinação da atividade antimicrobiana (Araújo, 2003; Souza et al., 2004a;
Lima et al., 2005; 2006).

A atividade antifúngica do óleo essencial de alecrim já foi descrita por outros estudos (Sacchete et al., 2005; Lima et al., 2006; Pozzatti et al., 2008; Van Vuuren et al., 2009), havendo, porém, variabilidade quanto ao tipo de microrganismos e técnica metodológica utilizada. Porém, a atividade antifúngica do óleo essencial do sassafrás foi pouco estudada, justificando, assim, a realização deste estudo, que busca contribuir no sentido de fornecer dados para a literatura científica.

Assim, foi propósito desse estudo avaliar a susceptibilidade de espécies de $C$. albicans e $C$. tropicalis frente ao óleo essencial de Ocotea odorifera Vell. e Rosmarinus officinalis $\mathrm{L}$.

\section{MATERIAL E MÉTODO}

\section{Óleos essenciais}

Os óleos essenciais que tiveram a atividade antifúngica avaliada foram obtidos na Ferquima Ind. e Com. Ltda (Vargem Grande Paulista, São Paulo, Brasil), sendo seus parâmetros físico-químicos descritos pelo fornecedor, que produz e comercializa óleos essenciais em escala industrial, como ilustrado na Tabela 1.

TABELA 1. Óleos essenciais avaliados para determinação da atividade antifúngica, João Pessoa-PB, 2009.

\begin{tabular}{c|c|c|c|l|l}
\hline Espécie & Família & $\begin{array}{c}\text { Nome } \\
\text { popular }\end{array}$ & $\begin{array}{c}\text { Parte } \\
\text { utilizada }\end{array}$ & $\begin{array}{c}\text { Constituinte (s) } \\
\text { químico (s) }\end{array}$ & \multicolumn{1}{|c}{ Refe rência } \\
\hline Ocotea odorifera Vell. & Lauraceae & Sassafrás & Folhas & Safrol & $\begin{array}{l}\text { Barreiro \& Fraga, } \\
1999\end{array}$ \\
\hline Rosmarinus officinalis L. & Lamiaceae & Alecrim & Folhas & $\begin{array}{l}\text { a-pinene, } \beta \text {-mirceno, } \\
\text { cânfora eucaliptol }\end{array}$ & $\begin{array}{l}\text { Angioni et al., } \\
2004 ; \text { Pris et al., } \\
2006\end{array}$ \\
\hline
\end{tabular}

\section{Espécies fúngicas}

Foram incluídas no estudo 16 cepas fúngicas: C. albicans ATCC-90028, C. albicans ATCC-76615, C. albicans ATCC-76645, C.albicans ATCC-76485, C. tropicalis ATCC-13803, C. albicans LM-42V, C. albicans18F, C. albicans MD-37, C. albicans LM-968, C. albicans ICB-12, C. tropicalis LM-708, C. tropicalis LM-14, C. tropicalis LM-028, C. tropicalis LM-37, C. tropicalis LM-13 e C. tropicalis LM-759.

As cepas foram mantidas em ágar Sabouraud dextrose -ASD a temperatura de $4^{\circ} \mathrm{C}$, sendo utilizados para os ensaios repiques de 24 horas em ASD incubados a $35^{\circ} \mathrm{C}$. No estudo da atividade antimicrobiana utilizou-se inóculo fúngico de, aproximadamente, $10^{6}$ UFC mL-1 padronizado de acordo com a turbidez do tubo 0,5 da escala de McFarland.

\section{Diluição dos óleos essenciais de 0 . odorifera e $R$. officinalis}

Os óleos essenciais de $O$. odorifera e $R$. officinalis foram diluídos, separadamente, seguindo as recomendações de Allegrini et al. (1973), que consiste na adição de $0,4 \mathrm{~mL}$ do óleo essencial, 0,04 $\mathrm{mL}$ de TWEEN 80 e água destilada estéril q.s.p. 5 $\mathrm{mL}$. A mistura foi mantida sob constante agitação, por cinco minutos, em aparelho Vortex.

\section{Determinação da Concentração Inibitória Mínima (CIM) - Técnica da Microdiluição}

A determinação da CIM para os óleos essenciais foi realizada através da técnica da microdiluição, com algumas adaptações, preconizada pelo Método de Referência para Testes de Diluição em Caldo para a Determinação da Sensibilidade de 
Leveduras à Terapia Antifúngica (NCCLS, 2002). Inicialmente, foram distribuídos $100 \mu \mathrm{L}$ de caldo Sabouraud dextrose nos orifícios das placas de microdiluição. Em seguida, foram distribuídos $100 \mu \mathrm{L}$ da emulsão do óleo essencial, a uma concentração inicial de $5 \mathrm{mg} \mathrm{mL}^{-1}$, que foi diluída seriadamente, à partir da retirada de uma alíquota de $100 \mu \mathrm{L}$ da cavidade mais concentrada para a cavidade sucessora, gerando uma concentração final de $0,039 \mathrm{mg} \mathrm{mL}^{-1}$. Nos orifícios de cada coluna foram dispensadas alíquotas de $10 \mu \mathrm{L}$ do inóculo correspondente a cada cepa ensaiada na

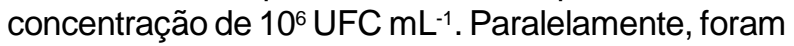
realizados controle da viabilidade das cepas de leveduras ensaiadas. Realizou-se também teste de sensibilidade das cepas ensaiadas frente à ação dos antifúngicos miconazol e nistatina, consideradas drogas de escolha na utilização clínica, em concentrações iniciais de $32 \mu \mathrm{g} \mathrm{mL}^{-1}$ e $64 \mu \mathrm{g} \mathrm{mL}-1$, respectivamente. Para controle de qualidade da metodologia empregada, foi realizado o teste de esterilidade do meio de cultura empregado.

Os ensaios foram realizados em duplicata e incubados a $35^{\circ} \mathrm{C}$ durante $24-48$ horas. A leitura para determinação da CIM dos óleos essenciais sobre as cepas de leveduras foi feita a partir do método visual. Foi levada em consideração a formação ou não de aglomerados de células ("crescimento visual") no fundo da cavidade da placa. Dessa forma, considerouse como CIM, a menor concentração do produto em teste capaz de produzir inibição visível sobre o crescimento das cepas de leveduras utilizadas nos ensaios microbiológicos (NCCLS, 2002).

Para confirmação da presença de microrganismos viáveis nas concentrações não inibitórias, foi utilizado o corante TCC $(2,3,5$ trifenil cloreto de tetrazólio) no volume de $10 \mu \mathrm{L}$, que reflete a atividade das enzimas desidrogenases, envolvidas no processo de respiração. Isto torna possível distinguir as amostras vivas, coloridas de vermelho, daquelas mortas que mantêm a sua cor (Gabre, 1976; Deswal \& Chand, 1997).

\section{RESULTADO}

Todas as cepas ensaiadas, com exceção da C. tropicalis LM 708, apresentaram-se sensíveis ao óleo essencial de $O$. odorífera, sendo observado uma CIM de $2,5 \mathrm{mg} \mathrm{mL}^{-1}$ sobre $68 \%$ das cepas utilizadas

TABELA 2. Valores de CIM ( $\left.\mathrm{mg} \mathrm{mL}^{-1}\right)$ dos óleos essenciais de $O$. odorifera Vell. e $R$. officinalis L. frente às cepas de Candida albicans e C. tropicalis.

\begin{tabular}{|c|c|c|c|c|}
\hline Espécies fúngicas & $\begin{array}{c}\mathrm{CIM}\left(\mathrm{mg} \mathrm{mL}^{-1}\right) \\
\text { O. odorifera Vell. }\end{array}$ & $\begin{array}{l}\mathrm{CIM}\left(\mathrm{mg} \mathrm{mL}^{-1}\right) \\
\text { R. officinalis } \mathrm{L} \text {. }\end{array}$ & $\begin{array}{c}\text { CIM }\left(\mu \mathrm{g} \mathrm{mL^{-1 }}\right) \\
\text { Nistatina }\end{array}$ & $\begin{array}{c}\mathrm{CIM}\left(\mu \mathrm{g} \mathrm{mL} \mathrm{m}^{-1}\right) \\
\text { Miconazol }\end{array}$ \\
\hline C. albicans ATCC-90028 & 2,5 & 2,5 & 2 & 1 \\
\hline C. albicans ATCC- 76615 & 2,5 & 2,5 & 4 & 2 \\
\hline C. albicans ATCC- 76645 & 2,5 & 2,5 & 4 & 1 \\
\hline C. albicans ATCC- 76485 & 2,5 & 2,5 & 4 & 1 \\
\hline C. albicans LM- $42 \mathrm{~V}$ & 2,5 & $>5$ & 8 & 2 \\
\hline C. albicans $18 \mathrm{~F}$ & 2,5 & 2,5 & 2 & 1 \\
\hline C. albicans MD-37 & 5 & 5 & 4 & 1 \\
\hline C. albicans LM-968 & 5 & 2,5 & 4 & 1 \\
\hline C. albicans ICB-12 & 2,5 & $>5$ & 2 & 1 \\
\hline C. tropicalis ATCC-13803 & 5 & 5 & 1 & 0,5 \\
\hline C. tropicalis LM-708 & $>5$ & $>5$ & 4 & 1 \\
\hline C. tropicalis LM -759 & 2,5 & 5 & 4 & 1 \\
\hline C. tropicalis LM-14 & 2,5 & 5 & 4 & 1 \\
\hline C. tropicalis LM-28 & 2,5 & 5 & 4 & 2 \\
\hline C. tropicalis LM-37 & 2,5 & 5 & 4 & 1 \\
\hline C. tropicalis LM-13 & 5 & 5 & 4 & 0,5 \\
\hline
\end{tabular}

Rev. Bras. PI. Med., Botucatu, v.13, n.2, p.203-208, 2011. 
nos ensaios microbiológicos. Resultado semelhante foi observado para a $R$. officinalis L., onde foi encontrada uma CIM de $5 \mathrm{mg} \mathrm{mL}^{-1}$ sobre $81 \%$ das cepas ensaiadas, sendo identificada resistência apresentada pela $C$. albicans LM42V, C. albicans ICB12 e C. tropicalis LM078 (Tabela 1).

Todas as cepas mostraram-se sensíveis aos produtos sintéticos padrões avaliados, miconazol e nistatina, utilizados em concentrações de $32 \mu \mathrm{g} \mathrm{mL}^{-1}$ e $64 \mu \mathrm{g} \mathrm{mL}^{-1}$, respectivamente. Foram observadas CIMs de $4 \mu \mathrm{g} \mathrm{mL}^{-1}$ e $1 \mu \mathrm{g} \mathrm{mL}^{-1}$ de miconazol e nistatina, respectivamente, para a C. albicans ATCC 90028. Para a cepa de C. tropicalis ATCC 13803, miconazol e nistatina em concentrações de $2 \mu \mathrm{g} \mathrm{mL}^{-1}$ e $1 \mu \mathrm{g} \mathrm{mL}^{-1}$, respectivamente, foram capazes de inibir o crescimento.

\section{DISCUSSÃO}

Com frequencia, as infecções fúngicas, incluindo as da cavidade oral, são de difícil tratamento, fato que está intrinsecamente relacionado à aquisição por parte dos seus agentes etiológicos de resistência à ação de antifúngicos disponíveis. Nesse sentido, utilizar produtos de origem natural para tratamento de tais infecções representa importante possibilidade de tratamento.

Óleos essenciais obtidos a partir de plantas são considerados fontes promissoras para elaboração de fármacos utilizados no tratamento de micoses, mesmo considerando que os fungos envolvidos em infecções humanas apresentam-se relativamente mais sensíveis aos antifúngicos sintéticos comerciais (Faleiro et al., 2003; Shin \& Pyun, 2004).

A atividade antifúngica do óleo essencial de Rosmarinus officinalis $\mathrm{L}$. tem sido objeto de estudo de várias pesquisas (Panizzi et al., 1993; Larrondo et al., 1995; Mangena \& Muyima, 1999; Daferera et al., 2000; Lorenzi \& Matos, 2002; Fenner et al., 2006). Simões et al. (1998) relatam que os óleos voláteis da família Lamiaceae, a qual pertence $R$. officinalis L., apresentam grande importância econômica e várias espécies são cultivadas para utilização na indústria de alimentos, cosméticos e, também, para fins medicinais.

Nascimento et al. (2000) verificaram susceptibilidade da $C$. albicans frente ao extrato hidroalcoólico de $R$. officinalis L. Quanto ao óleo essencial, Packer \& Luz (2007) observaram, a partir do teste de difusão em meio sólido, atividade fungistática do mesmo, identificando halos de inibição maiores que $60 \mathrm{~mm}$.

Corroborando com os resultados desta pesquisa, Lima et al. (2006) verificaram incipiente atividade antifúngica do óleo essencial do $R$. officinalis L. a $8 \%$, sendo observado halo de inibição de $10 \mathrm{~mm}$ para a cepa de C. albicans ATCC 76615 e ausência de atividade para cepas de $C$. tropicalis. Resultados semelhantes também foram observados por Araújo et al. (2004), que avaliaram a atividade antifúngica frente cepas de Candida do óleo essencial dessa planta.

Quanto à atividade da Ocotea odorífera Vell., Souza et al. (2004a) verificaram resistência da Candida albicans ATCC10231 frente ao extrato hidroalcoólico. Em outro estudo, realizado por Souza et al. (2004b), também foi observada ausência de atividade antimicrobiana da Ocotea odorífera Vell. Ressalta-se que estudos envolvendo a atividade antifúngica do óleo essencial são escassos, sendo esta pesquisa pioneira na determinação desta.

Como esperado, os antifúngicos sintéticos utilizados como controle do estudo (miconazol e nistatina) apresentaram satisfatória atividade sobre as cepas ensaiadas, com CIMs variando entre $1 \mu \mathrm{g}$ $\mathrm{mL}^{-1}$ e $4 \mu \mathrm{g} \mathrm{mL} \mathrm{m}^{-1}$. Tal resultado tem justificado a utilização dessas substâncias como primeira escolha no tratamento de candidose oral (Batista et al., 1999).

A fraca atividade antifúngica frente cepas de Candida apresentada pelos óleos essenciais utilizados neste estudo pode ser explicada pelas características químicas dos mesmos, como volatilidade, insolubilidade em água e complexidade, que podem interferir significativamente nos resultados (Nascimento et al., 2007). Por outro lado, a hidrofobicidade apresentada pelos óleos essenciais, responsável pelo provável mecanismo de ação, pode facilitar sua interação com estruturas celulares que tem constituição lipídica, promovendo aumento da permeabilidade, provocando uma saída extensiva de eletrólitos, indispensáveis à sobrevivência celular (Prabuseenivasan et al., 2006).

Como descrito por Celiktas et al. (2007), as condições ambientais, como solo, umidade e temperatura, podem influenciar na atividade antimicrobiana dos óleos essenciais. Porém, como os óleos avaliados neste estudo foram obtidos em estabelecimento comercial, não se conhece as reais condições de coleta e processamento do material botânico, indicando, assim a necessidade de realização de novos estudos.

A partir dos dados obtidos foi possível concluir que os óleos essenciais de Ocotea odorífera Vell. e Rosmarinus officinalis $L$. apresentam fraca atividade sobre espécies fúngicas envolvidas em infecções da cavidade oral. Nesse sentido, faz-se necessária a realização de outros ensaios microbiológicos com vista a elucidar a atividade antifúngica dos óleos essenciais de Ocotea odorífera Vell. e Rosmarinus officinalis L., bem como dos fitoconstituintes, na perspectiva de elaboração de fármaco seguro e eficaz para tratamento de micoses superficiais da cavidade oral. 


\section{REFERÊNCIA}

ALLEGRINI, J.; BOUCHBERG, M.S.; MAILOLS, H. Émulsiond d'huilles essentielles fabricaton et applications en microbiologie. Society Pharmaceutical Montpellier, v.33, p.73-86, 1973.

ANGIONI, A. et al. Chemical composition, plant genetic differences, antimicrobial and antifungal activity investigation of the essential oil of Rosmarinus officinalis L. Journal of Agricultural and Food Chemistry, v.52, n.11, p.3530-5, 2004.

ARAÚJO, J.C.L.V. Perfil de sensibilidade de microrganismos oportunistas de origem clínica e ambiental a óleos essenciais. 2003. 77p. Dissertação (Mestrado em Produtos Naturais e Sintéticos Bioativos)Departamento de Ciências Farmacêuticas, Universidade Federal da Paraíba, João Pessoa.

ARAÚJO, J.C.L.V. et al. Ação antimicrobiana de óleos essenciais sobre microrganismos potencialmente causadores de infecções oportunistas. Revista de Patologia Tropical, v.33, n.1, p.55-64, 2004.

BARREIRO, E.J.; FRAGA, C.A.M. A utilização do safrol, principal componente químico do óleo de sassafráz, na síntese de substâncias bioativas na cascata do ácido araquidônico: antiinflamatórios, analgésicos e antitrombóticos. Química Nova, v.22, n.5, p.744-59, 1999.

BATISTA, J.M.; BIRMAN, E.G.; CURY, A.E. Suscetibilidade a antifúngicos de cepas de Candida albicans isoladas de pacientes com estomatite protética. Revista de Odontologia da Universidade de São Paulo, v.13, n.4, p.343-8, 1999.

CELIKTAS, O.Y. et al. Antimicrobial activities of methanol extracts and essential oils of Rosmarinus officinalis, depending on location and seasonal variations. Food Chemistry, v.1, n.100, p.553-9, 2007.

DAFERERA, D.J.; ZIOGAS, B.N.; POLISSIOU, M.G. Analysis of essential oils from some Greek aromatic plants and their fungitoxicity on Penicillium digitatum. Journal of Agricultural and Food Chemistry, v.48, n.1, p.2576-81, 2000.

DESWAL, D.P.; CHAND, U. Standartization of the tetrazolium test for viability estimation in ricebean (Vigna umbellate T.) seeds. Seed Science and Technology, v.25, n.1, p.409-17, 1997.

FALEIRO, M.L. et al. Antimicrobial activity of essential oils isolated from Portuguese endemic species of Thymus. Letters in Applied Microbiology, v.29, n.1, p.130-5, 2003. FENNER, R. et al. Plantas utilizadas na medicina popular brasileira com potencial atividade antifúngica. Revista Brasileira de Ciências Farmacêuticas, v.42, n.3, p.36994, 2006.

GABRE, D. F. Manual do teste de tetrazólio. Brasília: AGIPLAN, 1976. 85p.

HENRIQUES, M.; AZEREDO, J.; OLIVEIRA, R. Adhesion of Candida albicans and Candida dubliniensis to acrylic and hydroxyapatite. Biointerfaces, v.33, n.1, p.235-41, 2004.

LARRONDO, J.V.; AGUT, M.; CALVO-TORRAS, M.A. Antimicrobial activity of essences from labiates. Microbios, v.82, n.1, p.171-2, 1995.

LIMA, E.O.; SOUZA, E.L.; LIMA, I.O. Espectro antimicrobiano do óleo essencial de Rosmarinus officinalis L. Revista Brasileira de Ciências da Saúde, v.9, n.1, p.17-24, 2005.
LIMA, I.O. et al. Atividade antifúngica de óleos essenciais sobre espécies de Candida. Revista Brasileira de Farmacognosia, v.16, n.2, p.197-201, 2006.

LORENZI, H.E.; MATOS, F.J. Plantas medicinais no Brasil: nativas e exóticas. Nova Odessa: Instituto Plantarum. 2002. p.512.

MANGENA, T.; MUYIMA, N.Y. Comparative evaluation of the antimicrobial activities of essences oils of Artemisia afra, Pteronia incana and Rosmarinus officinalis selected bacteria and yeast strains. Letters in Applied Microbiology, v.28, n.1, p.291-6, 1999.

MONGE, R.A. et al. The MAP Kinase signal transduction network in Candida albicans. Microbiology, v.152, n.1, p.905-12, 2006.

MÜLLER, V. et al. Candida albicans triggers activation of distinct signaling pathways to establish a proinflammatory gene expression program in primary human endothelial cells. The Journal of Immunology, v.179, n.1, p.8435-45, 2007.

NASCIMENTO, G.G.F. et al. Antibacterial activity of plant extracts and phytochemicals on antibiotic-resistant bacteria. Brazilian Journal of Microbiology, v.31, n.1, p.247-56, 2000.

NASCIMENTO, P.F.C. et al. Atividade antimicrobiana dos óleos essenciais: uma abordagem multifatorial dos métodos. Revista Brasileira Farmacognosia, v.17, n.1, p.108-13, 2007.

NATIONAL COMMITTEE FOR CLINICAL LABORATORY STANDARD - NCCLS. Método de referência para testes de diluição em caldo para determinação da sensibilidade de leveduras à terapia antifúngica: norma aprovada - M27-A2. 2.ed. Wayne, 2002.

OROZCO, A.S.; ZHOU, X.; FILLER, S.G. Mechanisms of the proinflammatory response of endothelial cells to Candida albicans infection. Infection and Immunity, v.68, n.3, p.1134-41, 2000.

PACKER, J.F.; LUZ, M.M.S. Método para avaliação e pesquisa da atividade antimicrobiana de produtos de origem natural. Revista Brasileira de Farmacognosia, v.17, n.1, p.102-7, 2007.

PANIZZI, L. et al. Composition and antimicrobial properties of essential oils of four Mediterranean Lamiaceae. Journal of Ethnopharmacology, v.39, n.1, p.167-70, 1993.

PRABUSEENIVASAN, S.; JAYAKUMAR, M.; IGNACIMUTHU, S. In vitro antibacterial activity of some plant essential oils. BMC Complementary and Alternative Medicine, v.6, n.39, p.1-8, 2006.

POZZATTI, P. et al. In vitro activity of essential oils extracted from plants used as spices against fluconazole-resistant and fluconazole-susceptible. Canadian Journal of Microbiology, v.54, n.1, p.950-6, 2008.

PRIS, C.L.; LEMOS, C.S.L; FREITAS, S.P. Efeito do tempo de extração sobre a composição e rendimento do óleo essencial de Alecrim (Rosmarinus officinalis). Revista Brasileira de Plantas Medicinas, v.8, n.4, p.92-5, 2006. RAMAGE, G. et al. Candidabiofilms: an update. Eukaryotic Cell, v.4, n.4, p.633-8, 2005.

SACCHETTI, G. et al. Comparative evaluation of 11 essential oils of diferent origin as functional antioxidants, antiradicals and antimicrobials in foods. Food Chemistry, v.91, n.4, p.621-32, 2005.

SHIN, S.; PYUN, M. Anti-Candida effects of estragole in 
combination with ketoconaloze or amphotericin B. Phhytotherapy Research, v.18, n.1, p.827-30, 2004.

SIMÕES, C.M.O. et al. Plantas da medicina popular do Rio Grande do Sul. 5.ed. Porto Alegre/Florianópolis: Editora UFRGS/UFSC, 1998. 173p.

SOUZA, G.C. et al. Ethnopharmacological studies of antimicrobial remedies in the south of Brazil. Journal of Ethnopharmacology, v.90, n.1, p.135-43, 2004a.

SOUZA, G.C. et al. Community home pharmacies in Maquine (RS): an ethnopharmacological evaluation. Revista Brasileira de Plantas Medicinais, v.6, n.2, p.8391, 2004b.
THEIN, Z.M.; SAMARANAYAKE, Y.H.; SAMARANAYAKE, L.P. Effect of oral bacteria on growth and survival of Candida albicans biofilms. Archives of Oral Biology, v.51, n.1, p.672-80, 2006.

VAN VUUREN, S.F.; SULIMAN, S.; VILJOEN, A.M. The antimicrobial activity of four commercial essential oils in combination with conventional antimicrobials. Letters in applied microbiology, v.48, n.4, p.440-6, 2009. WINGETER, M.A. et al. Identificação microbiológica e sensibilidade in vitro de Candida isoladas da cavidade oral. Revista da Sociedade Brasileira de Medicina Tropical, v.40, n.3, p.272-6, 2007. 